\section{Minimal Mean-Square Error for 3D MIMO Beamforming Weighting}

\section{Cheng Xu, John Cosmas and Yue Zhang}

The 3D MIMO beamforming system needs a weighting method to determine the direction of beam whist reducing the interference for other beam areas operating at the same carrier frequency. The challenge is to determine the weights of the 3D MIMO beams to direct each beam towards its cluster of user terminals whilst placing its nulls at undesired user directions in order to minimise undesired interference. Therefore, the SINR (signal to interference plus noise ratio) should be increased whilst the interference from the side lobes of the other beams reduced. A weight determining method is presented in this paper that constructs horizontal and vertical array weights respectively by MMSE (minimizing the mean-square error) between the array pattern vector and the unit vector, where the unit vector expresses the desired direction for the array pattern and zero vector expresses the undesired direction. Since the rectangular planar array can be viewed as M linear arrays of $\mathrm{N}$ elements, the weight of the M-Nth element can be obtained based on the horizontal and vertical array weights.

Introduction: The 3D MIMO beamforming is one of the key technologies for 5G system, which has a fundamental dichotomy. The system can be configured to either service a group of users in its coverage area with a wide beamforming radiation pattern and a wide bandwidth transmission frame or it can be configured to service a single user in its coverage area with a narrow beamforming radiation pattern and a narrow bandwidth. It is envisaged that 3D MIMO will operate in millimetre wave frequencies with very wide bandwidth so a narrow beamforming for a single user cannot efficiently utilise the whole transmission frame capacity. There are two considerations to solve. The first is how many users should be covered by one 3D beamforming, which will determine how many dipole antennas will be used to transmit the 3D MIMO signal. The K-means algorithm is a good scheme to solve this problem by grouping the user terminals into clusters that can be serviced by the 3D beamforming. The second is the range of SINR for the different user terminals in the main beam after the K-means algorithm has grouped the user terminals into different clusters.

K-means algorithm: The basic concept of clustering is to divide patterns into different groups (clusters). Amongst many different clustering algorithms, K-means algorithm is a better algorithm for clustering a low dimensional data set $[1,2]$. In K-means algorithm, the user terminals are divided into several groups based on the vertical and horizontal angles between user terminals. If the angles between a user terminal and one cluster centre are the smallest among the angles to other cluster centres, then this user terminal will belong to the cluster corresponding to this cluster centre. It is well-known that K-means algorithm only divides users into $\mathrm{K}$ different groups, which means that every user in one group is covered by one beam. For example, the cell will be separated into 6 areas and the user terminals are randomly distributed within each area. Then every area will be divided into 2 parts, which is based on the height of user terminals. Then the K-means algorithm is used to determine the cluster centre for each group. Fig. 1 shows one area that has 6 user terminals, which are covered by 3 beams.

\section{There are 3 Beams and 6 Users in the 4 th Face for the 9th Simulation}

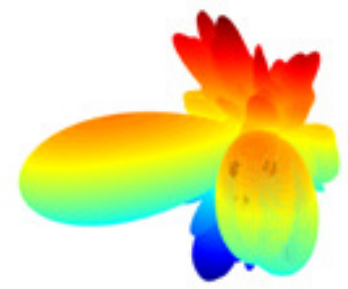

Fig. 1 Three main beams for user terminals in one area

In Fig. 1, the three main lobes have an effect on each other that is measured in SINR and the antenna array weights for parameterising them should be set to minimise the interference. In this paper, the optimum antenna array weights are determined, which steers the beam towards its cluster of user terminals whilst maximizing the SINR

Determining the weight of each array element: For a rectangular array in the $\mathrm{x}$-y plane, there are $\mathrm{M}$ elements in the $\mathrm{x}$-direction and $\mathrm{N}$ elements in the $y$-direction. The $\mathrm{x}$-directed elements are spaced $\mathrm{dx}$ apart and the $y$-directed elements are spaced dy apart. According to [3], we obtain the pattern of the entire $\mathrm{M} \times \mathrm{N}$ element array:

$$
\begin{aligned}
& A F=A F_{x} \cdot A F_{y} \\
& =\sum_{m=1}^{M} a_{m} e^{j(m-1)\left(k d_{x} \sin \theta \cos \varphi+\beta_{x}\right)} \sum_{n=1}^{N} b_{n} e^{j(n-1)\left(k d_{y} \sin \theta \sin \varphi+\beta_{y}\right)}
\end{aligned}
$$

In order to allow the desired signal to be received without modification and reject the undesired interfering signals, let $\mathrm{AF}=1$ in the desired direction and $\mathrm{AF}=0$ in the undesired interfering direction. That is in the desired direction, we hope

$$
A F_{x}=1 \text { and } A F_{y}=1
$$

in the undesired direction, we hope

$$
A F_{x}=0 \text { and } A F_{y}=0
$$

(1) Calculating the weight vector in $\mathrm{x}$-direction $W_{x}$

$$
\begin{aligned}
& \text { Let } W_{x}=\left[\begin{array}{llll}
a_{1} & a_{2} & \ldots & a_{M}
\end{array}\right]^{T} \text {, then } \\
& A F_{x}=\sum_{m=1}^{M} a_{m} e^{j(m-1)\left(k d_{x} \sin \theta \cos \varphi+\beta_{x}\right)} \\
& =\left[\begin{array}{llll}
1 & e^{j\left(k d_{x} \sin \theta \cos \varphi+\beta_{x}\right)} a_{2} & \ldots & e^{j(m-1)\left(k d_{x} \sin \theta \cos \varphi+\beta_{x}\right)}
\end{array}\right] W_{x}
\end{aligned}
$$

In our paper, if there are four beams in one area, then the one desired direction will be $\theta=\theta_{0}$ and $\varphi=\varphi_{0}$; three undesired direction are respectively:

$$
\begin{aligned}
& \theta=\theta_{1} \text { and } \varphi=\varphi_{1} \\
& \theta=\theta_{2} \text { and } \varphi=\varphi_{2} \\
& \theta=\theta_{3} \text { and } \varphi=\varphi_{3}
\end{aligned}
$$

In the desired direction, from (1), we have

$$
\left[\begin{array}{llll}
1 & e^{j\left(k d_{x} \sin \theta_{0} \cos \varphi_{0}+\beta_{x}\right)} & \ldots & e^{j(m-1)\left(k d_{x} \sin \theta_{0} \cos \varphi_{0=}+\beta_{x}\right)}
\end{array}\right] W_{x}=1(2)
$$

In undesired direction, from (1), we have

$$
\begin{aligned}
& 1 \quad e^{j\left(k d_{x} \sin \theta_{1} \cos \varphi_{1}+\beta_{x}\right)} a_{2} \quad \ldots \quad e^{j(m-1)\left(k d_{x} \sin \theta_{1} \cos \varphi_{1=}+\beta_{x}\right)} \mid W_{x}=0 \text { (3) } \\
& {\left[\begin{array}{llll}
1 & e^{j\left(k d_{x} \sin \theta_{2} \cos \varphi_{2}+\beta_{x}\right)} a_{2} & \ldots & e^{j(m-1)\left(k d_{x} \sin \theta_{2} \cos \varphi_{2}+\beta_{x}\right)}
\end{array} \mid W_{x}=0\right. \text { (4) }} \\
& {\left[\begin{array}{llll}
1 & e^{j\left(k d_{x} \sin \theta_{3} \cos \varphi_{3}+\beta_{x}\right)} a_{2} & \ldots & e^{j(m-1)\left(k d_{x} \sin \theta_{3} \cos \varphi_{32}+\beta_{x}\right)}
\end{array}\right] W_{x}=0 \text { (5) }}
\end{aligned}
$$

According to (2)-(5), we obtain

$$
\left[\begin{array}{cccc}
1 & e^{j\left(k d_{x} \sin \theta_{0} \cos \varphi_{0}+\beta_{x}\right)} & \ldots & e^{j(m-1)\left(k d_{x} \sin \theta_{0} \cos \varphi_{0=}+\beta_{x}\right)} \\
1 & e^{j\left(k d_{x} \sin \theta_{1} \cos \varphi_{1}+\beta_{x}\right)} & \ldots & e^{j(m-1)\left(k d_{x} \sin \theta_{1} \cos \varphi_{1=}+\beta_{x}\right)} \\
1 & e^{j\left(k d_{x} \sin \theta_{2} \cos \varphi_{2}+\beta_{x}\right)} & \ldots & e^{j(m-1)\left(k d_{x} \sin \theta_{2} \cos \varphi_{2=}+\beta_{x}\right)} \\
1 & e^{j\left(k d_{x} \sin \theta_{3} \cos \varphi_{3}+\beta_{x}\right)} & \ldots & e^{j(m-1)\left(k d_{x} \sin \theta_{3} \cos \varphi_{3}+\beta_{x}\right)}
\end{array}\right]\left[\begin{array}{c}
a_{1} \\
a_{2} \\
\ldots \\
a_{M}
\end{array}\right]=\left[\begin{array}{c}
1 \\
0 \\
0 \\
0
\end{array}\right]
$$

If we denote

$$
A=\left[\begin{array}{ccccc}
1 & e^{j\left(k d_{x} \sin \theta_{0} \cos \varphi_{0}+\beta_{x}\right)} & \ldots & e^{j(m-1)\left(k d_{x} \sin \theta_{0} \cos \varphi_{0=}+\beta_{x}\right)} \\
1 & e^{j\left(k d_{x} \sin \theta_{1} \cos \varphi_{1}+\beta_{x}\right)} & \ldots & e^{j(m-1)\left(k d_{x} \sin \theta_{1} \cos \varphi_{1=}+\beta_{x}\right)} \\
1 & e^{j\left(k d_{x} \sin \theta_{2} \cos \varphi_{2}+\beta_{x}\right)} & \ldots & e^{j(m-1)\left(k d_{x} \sin \theta_{2} \cos \varphi_{2=}+\beta_{x}\right)} \\
1 & e^{j\left(k d_{x} \sin \theta_{3} \cos \varphi_{3}+\beta_{x}\right)} & \ldots & e^{j(m-1)\left(k d_{x} \sin \theta_{3} \cos \varphi_{3}+\beta_{x}\right)}
\end{array}\right]
$$

and $b=\left[\begin{array}{llll}1 & 0 & \ldots & 0\end{array}\right]^{T}$, then $W_{x}$ is one solution of the following equation we have

$$
A X=b \quad(6)
$$

When $A$ is inversed, then $X=A^{-1} b$, however, $A$ is $4 \times M$ matrix.

In order to obtain the weight vector in $\mathrm{x}$-direction $W_{x}$, we need solve equation (6). Since $X=\operatorname{pinv}(A)^{*} b$ can minimize norm of vector $A X-b$, we can take $X=\operatorname{pinv}(A)^{*} b$ as an approximate solution of (6), that is

$$
W_{x}=\operatorname{pinv}(A) * b
$$

where pinv (A) is the Moore-Penrose pseudo inverse.

(2) In the same method, we can obtain the weight vector in ydirection $W_{y}$ 
(3) Calculating the weight matrix $W$

$$
W_{y}=\left[\begin{array}{llll}
b_{1} & b_{2} & \ldots & b_{N}
\end{array}\right]^{T}
$$

$$
W=W_{x}\left(W_{y}\right)^{T}=\left(w_{m n}\right)_{M \times N}
$$

where $w_{m n}$ is the weight of m-nth array element, and we have

$$
w_{m n}=a_{m} b_{n}
$$

Analysis and discussions on simulation results: For 3D MIMO the height of BS (base station) and UE will be taken into account during the simulation. The detailed simulation parameters are listed in Table1.

Table 1: The simulation configuration parameters

\begin{tabular}{|c|c|}
\hline Items & Parameters \\
\hline The times of the simulation & 20 \\
\hline Radius of base station $(\mathrm{km})(\mathrm{d} 2 \mathrm{D})$ & $0.2 \sim 5$ \\
\hline Height of the base station $(\mathrm{m})(\mathrm{hBS})$ & 25 or 150 \\
\hline Number of the antenna arrays & $16 \times 16$ \\
\hline $\begin{array}{c}\text { Number of antennasfor } \\
\text { beamforming }\end{array}$ & $8 \times 8$ \\
\hline The size of antenna element & Width 0.06, Length 0.06 \\
\hline Number of the move users & 10 \\
\hline Height of the move user $(\mathrm{m})(\mathrm{hUT})$ & $1.5 \sim 25$ \\
\hline Speed of motion & $3 \mathrm{~km} / \mathrm{h}$ \\
\hline The system scenarios & $3 \mathrm{D}-\mathrm{Uma}$ \\
\hline The carrier frequencies(GHz) & 2 \\
\hline $\begin{array}{c}\text { SubCarrier Frequency Spacing } \\
\text { (MHz) }\end{array}$ & 20 \\
\hline Thermal Noise $(\mathrm{dBm})$ & -174 \\
\hline Wavelength $(\mathrm{cm})$ & 11.99 \\
\hline The power of every antenna $(\mathrm{dBm})$ & 43 \\
\hline Base station & Gain 14dbi, Noise 5db \\
\hline Uesr terminal & Gain 0dbi, Noise 9db \\
\hline
\end{tabular}

In Fig.2 and Fig. 3, the CDF (Cumulative Distribution Function) of SINR is shown for 20 user terminals randomly distributed in one cell relative to the BS at heights 25 and 150 meters, respectively.

In order to make a better comparison with the proposed weight method, there are three methods to determine the weight of array element in our simulation analysis. The first is set each array element has the uniform weight. The second is the SVD algorithm from the channel information [4].The third one is our proposed MMSE method.

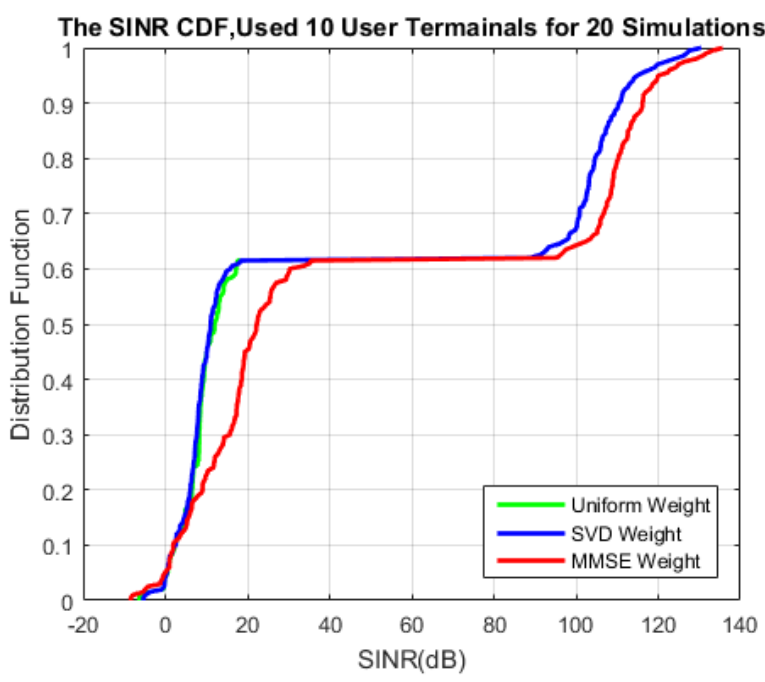

Fig. 2 SINR CDF for three methods to determine the weight when high of $B S$ is 25; green line shows that each array element has the uniform weight; blue line shows that the SVD algorithm is from the channel information; red line shows the MMSE weight method

In Fig, 2, the most of values for MMSE method are larger than $10 \mathrm{~dB}$ in red line, which is about $80 \%$. Moreover, the maximum value is more than $130 \mathrm{~dB}$. The values in the MMSE weight line are better than the values in Uniform Weight line and SVD Weight line. The proposed method has improved the SINR in 3D MIMO beamforming to clusters of user terminals.

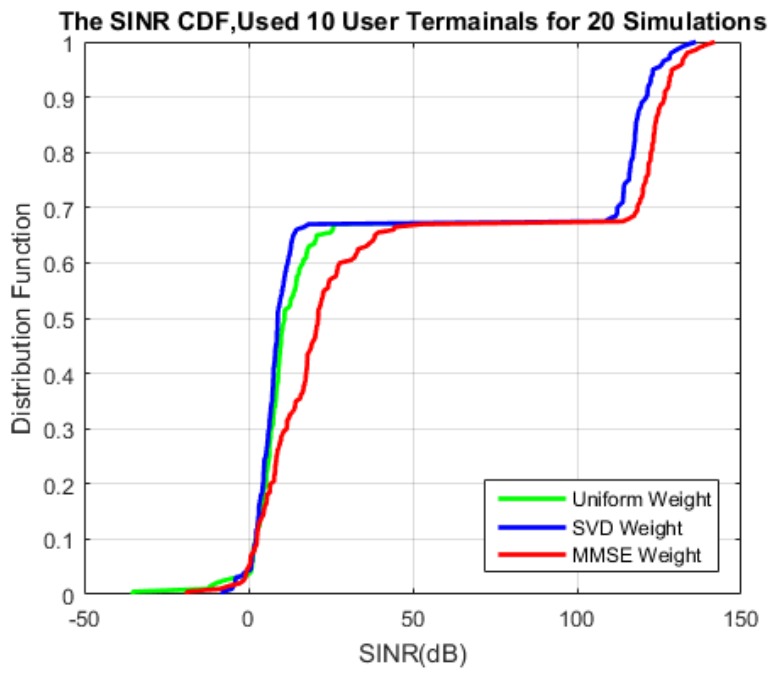

Fig. 3 SINR CDF for three methods to determine the weight when high of $B S$ is 150; green line shows that each array element has the uniform weight; blue line shows that the SVD algorithm is from the channel information; red line shows the MMSE weight method

In Fig. 3, $60 \%$ of the values in MMSE Weight line are smaller than $30 \mathrm{~dB}$, and corresponds with the performance observed in Fig. 2. Furthermore, the maximum value is more than $145 \mathrm{~dB}$, which is larger than the maximum value in Fig. 2. The performance results presented in Fig. 2 and Fig. 3 are compared in Table 2.

Table 2: The results for Fig.2 and Fig.3.

\begin{tabular}{|c|c|c|c|c|}
\hline \multirow{2}{*}{$\begin{array}{c}\text { Different } \\
\text { Weight }\end{array}$} & \multicolumn{2}{|c|}{ SINR in Fig.2 (25 m) } & \multicolumn{2}{c|}{ SINR in Fig.3 (150 m) } \\
\cline { 2 - 5 } & $\mathrm{CDF}=60 \%$ & $\mathrm{CDF}=40 \%$ & $\mathrm{CDF}=66 \%$ & $\mathrm{CDF}=34 \%$ \\
\hline $\begin{array}{c}\text { Green line } \\
(\mathrm{dB})\end{array}$ & $<20$ & $>90$ & $<15$ & $>110$ \\
\hline $\begin{array}{c}\text { Blue line } \\
(\mathrm{dB})\end{array}$ & $<20$ & $>90$ & $<25$ & $>110$ \\
\hline $\begin{array}{c}\text { Red } \\
\text { line(dB) }\end{array}$ & $<40$ & $>95$ & $<50$ & $>115$ \\
\hline
\end{tabular}

The Table 2 shows that the SINR values for the BS at $150 \mathrm{~m}$ height are larger than the values for BS at $25 \mathrm{~m}$ height. Therefore, the SINR performance will be better when the height of BS is higher.

When the location of user terminal is almost parallel to BS, the SINR will increase because the main-lobes will overlap each other.

To sum up, the proposed weight method has decreased the interference between each beam and identified that the location of BS should be set at the top of high building for increasing performance.

\section{References}

1. J. MacQueen, "Some methods for classification and analysis of multivariate observations", vol. 1 (Univ. of Calif. Press, 1967), pp. 281-297, Jan. 2008

2. Duda R O, Hart P E, "Pattern Classification and Scene Analysis", New York: Wiley-Interscience, 1973, pp. 482, received by Y. S. Chien, IEEE Transactions on Automatic Control, vol. 19, Jan. 2003

3. Frank B.Gross, "Smart Antennas for Wireless Communications with Matlab", McGraw-Hill Companies, 2005.

4. Yan Li, Xiaodong Ji, Dong Liang, and Yuan Li,"Dynamic beamforming for three-dimensional MIMO technique in LTEAdvanced networks", international Journal of Antennas and Propagation, 2013, pp.1-8. 